\title{
Economía de aglomeración y salarios en México. Un análisis en mercados laborales
}

\author{
Economy of agglomeration and wages in Mexico. \\ An analysis on labour markets
} \begin{abstract}
Resumen
En este trabajo se investiga como diversas
medidas relacionadas con las economías de aglomeración y de Nueva Geografía Económica (NGE) explican las diferencias salariales entre trabajadores manufactureros, utilizando como unidad de análisis los mercados laborales en México. Los resultados muestran que las variables de densidad y de diversidad económica, así como la distancia física al mercado americano son estadísticamente significativas y explican en más de $20 \%$ las diferencias de salario, aún después de controlar las características observables de los individuos. Estos hallazgos son robustos respecto a problemas de endogeneidad y dependencia espacial.
\end{abstract}

Palabras clave:

- Mercados laborales

- Economías de aglomeración

- Nueva Geografía Económica

\author{
Carlos Enrique Cardoso Vargas* :
}

\begin{abstract}
This paper investigates as various measures related to agglomeration economies and New Economic Geography (NEG) explain the wage gap between manufacturing workers, using as the analysis unit a labor markets in Mexico. The results show that the variables of density and economic diversity, as well as the physical distance to american market are statistically significant and explain more than $20 \%$ of the differences in wages, even after controlling for observable characteristics of individuals. These findings are robust to problems of endogeneity and spatial dependence.
\end{abstract}

\section{JEL: J31, R12, R12, J40}

\author{
Keywords: \\ - Labour Markets \\ - Economies of Agglomeration \\ - New Economic Geography
}

\section{Introducción}

En las últimas décadas México emprendió una serie ajustes estructurales y reformas económicas, particularmente relacionadas con hacer de ese país una economía más globalizada. La liberalización comercial emprendida por México ha tenido efectos diferenciados en la composición laboral y en la estructura salarial de las entidades federativas y en los sectores productivos. Está ampliamente documentado el incremento en la desigualdad salarial

\footnotetext{
* Investigador predoctoral en el Departamento de Economía Aplicada de la Universidad Autónoma de Barcelona. Edificio B Campus de la UAB Bellaterra (Cerdanyola del Vallès) C. P. 08193, Barcelona, España. Profesor-investigador de El Colegio del Estado de Hidalgo. Calle Miguel Hidalgo núm. 618, Colonia Centro, C. P. 42000, Pachuca, Hidalgo, México. Correo electrónico: CarlosEnrique.Cardoso@uab.cat y carlos.cardoso@elcolegiodehidalgo.edu.mx. El autor agradece los comentarios de José Luis Roig Sabaté. Este documento se enmarca dentro del proyecto ECO2010-20718 del Ministerio de Educación y Ciencias de España.
} 


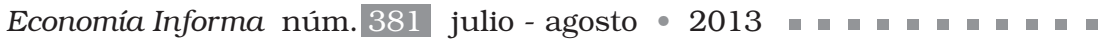

ocurrido a finales de los ochentas y principios de los noventas, que coincide con el inicio de la apertura comercial. ${ }^{1}$

En la literatura, las explicaciones sobre la desigualdad salarial que México registró durante esa etapa se han centrado en una mayor medida en dos hipótesis, el cambio técnico sesgado y las predicciones del Teorema StolperSamuelson (TSS). Los trabajos de Tan y Batra (1995), Cragg y Epelbaum (1996), Meza (1999), y Hanson y Harrison (1999), encuentran evidencia que soporta la hipótesis del cambio técnico sesgado. Por el contrario, la aproximación a través del TSS ha sido paradójica, ya que los resultados se encuentran en dos direcciones, los que no encuentran una relación sólida entre los cambios en el precio del producto y los salarios (Hanson y Harrison 1999, Feliciano 2001, y Cañonero y Werner, 2002) y los que encuentran evidencia del Teorema (Esquivel y Rodríguez-López 2003, Robertson 2004, y Chiquiar, 2008). Otros trabajos que han investigado los efectos de la liberalización comercial sobre los salarios son los de Hanson (1996, 1997 y 2003), quien ha encontrado que una parte significativa de los diferenciales salariales de México puede explicarse en función del acceso que tienen las regiones con respecto a los grandes mercados.

A nivel internacional existen diversos estudios empíricos que estudian las desigualdades salariales utilizando como marco de referencia a las teorías que explican la localización espacial de la actividad económica, como la Nueva Geografía Económica (NGE) y las economías de aglomeración. La mayor parte de esta literatura ha sido aplicada principalmente a países desarrollados, ${ }^{2}$ en cambio, la evidencia en países subdesarrollados es escasa. ${ }^{3}$

México representa un caso de interés, en virtud de que la apertura comercial propició, entre otras cosas, la relocalización de diversas industrias y

${ }^{1}$ Esta situación ha sido descrita en estudios que han utilizado diversas fuentes de información y diferentes coberturas de trabajadores. Entre los trabajos que dan cuenta del incremento de la desigualdad salarial entre empleados manufactureros utilizando los datos de la extinta Encuesta Nacional de Empleo, Salarios, Tecnología y Capacitación en el Sector Manufacturero (ENESTyC), son Feenstra y Hanson (1997); Hanson y Harrison (1999); y Revenga (1997). Por su parte, estudios que abarcan a los trabajadores de todos los sectores, se dividen en dos, los que usan la Encuesta Nacional de Empleo Urbano (ENEU) y los que utilizan la Encuesta de Ingreso Gasto de los Hogares. En el primer caso, encontramos los documentos de Cragg y Epelbaum (2004), y Robertson (2004); y en el segundo caso ubicamos el de Airola y Juhn (2005). ${ }^{2}$ Para una revisión de la literatura de NGE véase Head y Mayer (2004), y Redding (2010). Para en caso de Economía Urbana véase Rosenthal y Strange (2004).

${ }^{3}$ En este caso encontramos los trabajos de Fally et al. (2010) y Hering y Poncet (2009, 2010), que utilizando un marco teórico basado en la NGE investigan las desigualdades salariales en Brasil y China, respectivamente. Aunque los estudios de Hanson (1996, 1997 y 2003), aplicados a México, no se encuentran basado en un modelo de NGE, se relaciona con esta ya que considera en su evaluación la accesibilidad a los mercados. 
de factores de la producción (como la fuerza de trabajo), lo que se tradujo en modificaciones de las concentraciones económicas y demográficas del país. ${ }^{4}$ Estos cambios ocasionaron una nueva configuración de la geografía económica de México, tanto a nivel estatal como sectorial y representa un campo fértil para analizar el comportamiento de las desigualdades salariales desde la óptica de la NGE y las economías de aglomeración.

En este trabajo se investiga como diversas medidas relacionadas con las economías de aglomeración y de NGE explican las diferencias salariales entre trabajadores manufactureros de México. Para abordar este asunto usamos la información de los ingresos de los individuos varones que laboran en el sector manufacturero, la cual proviene de la muestra aleatoria de $1 \%$ proveniente del Censo de Población y Vivienda del año de 2000. Esta fuente de información tiene la ventaja, a diferencia de las encuestas de empleo, de abarcar una muestra más amplia de trabajadores, además, el uso de datos desagregados nos permite controlar en las estimaciones diversas características individuales de los trabajadores.

En las estimaciones se utiliza la metodología propuesta por Combes et al. (2008), que consiste en un procedimiento de dos etapas. En la primera los ingresos son regresionados con respecto a las características individuales observables de los trabajadores, controlando por efectos fijos área/ industria. En la segunda, los efectos fijos se regresionan con respecto a medidas de economías de aglomeración y de NGE. Esta metodología permite identificar de mejor manera el impacto de las medidas de utilizadas sobre los ingresos, ya que en la primera etapa descuenta los efectos de las características observables sobre la desigualdad en ingresos.

Asimismo, este documento incorpora dos elementos no considerados en la literatura que busca explicar la diferencia salarial en México. Primero, a diferencia de otros estudios que usan en sus estimaciones datos de estados o regiones, en nuestra estimación utilizamos como unidad espacial de análisis una reciente clasificación de mercados laborales locales realizada para México, que tiene un nivel geográfico menor al de los estados, pero superior a los municipios. Este aspecto es relevante, ya que el uso de unidad territorial construida con base en desplazamientos de los individuos por motivos de trabajo puede captar de mejor manera los efectos de la aglomeración sobre los salarios. Segundo, evaluamos la capacidad que tienen las medidas de la NGE y de las economías de aglomeración para explicar las diferencias

${ }^{4}$ Hanson (1997), muestra la existencia de una relocalización industrial del centro hacia al norte de México, posterior a la entrada en vigor del Tratado de Libre Comercio de América del Norte (TLCAN). 
salariales a distintas escalas territoriales, como entidades federativas y regiones.

Los resultados muestran que las variables de densidad y de diversidad económica asociadas con las economías de aglomeración, así como la medida de accesibilidad al mercado de Estados Unidos (relacionada con la NGE), son estadísticamente significativas y explican $24 \%$ de las diferencias de salario entre los mercados laborales, aún después de controlar las características observables de los trabajadores. Encontramos que la elasticidad de la densidad sobre los salarios se ubica en $2 \%$, en tanto que correspondiente a la diversidad y la medida de accesibilidad fue de 10 y $-9.1 \%$, respectivamente. Asimismo, estos hallazgos son robustos a otra forma de estimación, así como a problemas de endogeneidad y dependencia espacial. Cuando se evalúa el ámbito de influencia geográfico que tienen tales variables para explicar las diferencias salariales, se encuentra que las medidas de economías de aglomeración parecen explicar de mejor manera las diferencias en salarios en unidades geográficas menores (mercados laborales), por el contario la medida de accesibilidad parece ser más relevante en escalas espaciales superiores (estados y regiones).

$\mathrm{El}$ resto del documento esta ordenado de la siguiente manera. En el segundo apartado exponemos la literatura relacionada. En el tercero detalla la estrategia empírica y los datos utilizados. En los últimos dos se muestran estimaciones y finalmente las conclusiones.

\section{Literatura relacionada}

Muchos autores coinciden en que de manera general existen ventajas derivadas de una concentración de la actividad económica ${ }^{5}$ en términos de eficiencia para desempeñar diversas actividades económicas, sociales y políticas, que se justifican desde el punto de vista económico por la existencia de economías de escala. Estas ventajas son conocidas como economías de aglomeración, las cuales pueden generarse por una reducción de costos asociada al tamaño de planta, por la presencia de servicios especializados, la disponibilidad de infraestructuras, la presencia de un mercado laboral capacitado o la disponibilidad de un amplio mercado de bienes intermedios. ${ }^{6}$

Las economías de aglomeración se pueden clasificar en dos grandes categorías, economías de localización y economías de urbanización. Las pri-

\footnotetext{
${ }^{5}$ En esta línea se encuentran los trabajos de Marshall (1980), Weber (1909), Hirschman (1958) y Krugman (1991a), entre otros.

${ }^{6}$ Duranton y Puga (2004), consideran como fuentes de las economías de aglomeración al Sharing, Matching, y Learning.
} 
meras se refieren a los beneficios en productividad o reducción de costos que obtienen las empresas al estar cerca de otras firmas operando en el mismo sector o sectores relacionados. Estos beneficios derivan de la disponibilidad de un mercado laboral especializado, una amplia variedad de oferentes de bienes intermedios y de la posibilidad de compartir conocimiento entre las firmas. Las economías de urbanización son aquellas que consiguen las ganancias en productividad o disminución de costos por estar en un ambiente donde coexisten con firmas pertenecientes a industrias de todo tipo, tal entorno deriva de la presencia de un amplio mercado de trabajo, una abundante fuerza de trabajo diversificada, la existencia de amplias infraestructuras y bienes públicos.

Por su parte, la Nueva Geografía Económica (NGE) explica la concentración de la actividad económica tomando como la referencia la proximidad a grandes mercados lo que permite la reducción de costos de transporte, así como las ventajas de las externalidades derivadas de la difusión del conocimiento, las conexiones hacia delante y hacia atrás entre proveedores y productores, la existencia de mercados con trabajadores cualificados y la difusión de conocimiento (Krugman, 1991a y 1991b).

La idea atrás del uso de las economías de aglomeración y la NGE para explicar las desigualdades salariales se fundamenta en que si las empresas son más productivas en áreas donde se concentra más la actividad económica o tiene una mayor accesibilidad a los mercados, esas ubicaciones deberían reflejar salarios más altos. ${ }^{7}$

Existe una amplia literatura empírica respecto a las economías de aglomeración, principalmente aplicada países desarrollados. ${ }^{8}$ La conclusión de estos estudios es que doblando el tamaño de la ciudad parece incrementar la productividad entre industrias en un rango de 3 a 8\%. Henderson (1986) examina los efectos que tiene el tamaño de la industria local y de la economía local sobre la productividad laboral en industrias manufactureras en áreas urbanas de Estados Unidos (EU). Asimismo, revela que las grandes ciudades son más productivas debido a que estas tienen fuertes concentraciones de industrias específicas (economías de localización) y no porque estas fueran muy densas (economías de urbanización).

\footnotetext{
${ }^{7}$ En la economía neoclásica la productividad (producto marginal) de un trabajador tiene que ser al menos igual que su salario, porque concentraciones con firmas que registran altos niveles de productividad deberían también altos salarios.

${ }^{8}$ Rosenthal y Strange (2004), hacen una revisión extensa de dicha literatura. Muchos de estos estudios se centran en Estados Unidos, en una menor medida en Europa, y muy pocos abarcan a los países en desarrollo.
} 
Por su parte, Ciccone y Hall (1996) examinan empíricamente los efectos aglomeración sobre la productividad, encontrando que las economías de urbanización son significativas. Utilizando datos de productividad de las entidades federativas de Estados Unidos y la densidad del empleo como medida de aglomeración, muestran que un aumento de $10 \%$ en la densidad se traduce en un incremento de la productividad en 6\%. Además, concluyen que la densidad permite explicar en más de 50\% los diferenciales de productividad. En un estudio posterior, Ciccone (1997) con datos de los salarios medios de los condados de Estados Unidos observa que los efectos de la aglomeración estimados mediante el uso de los salarios son los mismos que los que se obtienen al trabajar con datos de productividad laboral.

Dentro de la literatura que ha abordado empíricamente la relación entre salarios y la accesibilidad a los mercados utilizando un marco teórico de NGE, destaca el trabajo pionero de Hanson (2005), ${ }^{9}$ quien con un panel de condados de Estados Unidos de los períodos de 1970-1980 y 1980-1990, concluye que existe una relación positiva entre los salarios y el acceso a mayores mercados, sin embargo, esta relación disminuye conforme la distancia a los consumidores aumenta. Con una metodología diferente Redding y Venables (2004), calculan impacto del acceso a los mercados (medido como potencial de mercado) sobre el PIB per cápita de 101 países a nivel internacional. En sus estimaciones encuentran evidencia de que los salarios son más altos en localizaciones centrales, con una alta demanda, que en áreas periféricas. También concluyen que la proximidad a los mercados y a los oferentes puede explicar en gran parte las diferencias en los salarios dentro de un país.

Mediante el uso de una estrategia distinta a las antes mencionadas, algunos estudios previos aplicados a México dan soporte a la relación entre salarios y potencial de mercado. Hanson (1996) muestra que los salarios relativos de la industria de prendas de vestir parecen ser más altos para aquellas entidades federativas que se encuentran más cerca del mercado estadounidense que para los ubicados al interior del territorio mexicano. Posteriormente, Hanson (1997) tomando utiliza las distancias lineales a la Ciudad de México y a Estados Unidos como aproximación de la accesibilidad a los mercados, encuentra que un aumento de $10 \%$ en la distancia con respecto a la Ciudad de México corresponde a una reducción de $1.9 \%$ en los salarios, mientras que un incremento de la misma magnitud con relación

${ }^{9}$ En esta línea se encuentran los estudios de Brakman et. al (2004) para Alemania, Mion (2004) para provincias Italianas y Neibuhr (2006) para regiones de 15 países de la Unión Europea, los cuales encuentran una relación positiva entre los salarios y el acceso a los mercados. 
a la distancia a la frontera norte ocasiona una disminución de $1.3 \%$ en los salarios.

En un trabajo reciente, Combes et al. (2008) estiman con un panel de datos de trabajadores franceses un modelo en el que se incorporan componentes utilizados en trabajos que evalúan las economías de aglomeración (densidad, área y la diversidad) y de la NGE (potencial de mercado), así como otras fuentes de disparidades salariales con la finalidad de conocer la importancia de esas explicaciones sobre las diferencias salariales en los mercados laborales de Francia. Estos autores concluyen que la composición de la fuerza laboral explica en gran parte de las diferencias salariales, una vez controladas las características no observables encuentran que el efecto de la densidad sobre los salarios es de $2 \%$, lo cual es mucho menor a lo detectado por la literatura previa.

\section{Estrategia empírica, datos y variables}

\section{1 Estimación en dos etapas}

La estrategia de estimación usada en este documento se basa en el procedimiento en dos etapas descrito en Combes et al. (2008). En la primera se estima el logaritmo de los salarios de los trabajadores con respecto a algunas características observables de los individuos (niveles de educación y experiencia) y efectos fijos área/industria. De esta forma, la ecuación a estimar es:

$$
\ln w_{l i j}=\beta_{0}+\beta_{1} e d a d_{1}+\beta_{2} e d a d_{l}^{2}+\beta_{3} e d u c_{l}+\beta_{3} f_{i j}+\varepsilon_{l i j}
$$

Donde $w_{l i j}$ es el ingreso del trabajador $l$ que labora la industria $i$ en el área $j$, la $\operatorname{edad}_{l}$ y $\operatorname{edad}_{l}^{2}$ capturan la experiencia del trabajador $l$, representa el vector que captura los niveles de educación del trabajador $l$ y $f_{i j}$ son los efectos fijos área/industria. En la segunda etapa se utilizan a los efectos fijos calculados como variable dependiente, los cuales representan un promedio de salario para cada área e industria. Estos efectos son regresionados con respecto a diversas medidas que se asocian con las economías de aglomeración y la NGE.

Las variables consideradas como parte de las economías de aglomeración son la densidad y la diversidad (economías de urbanización), así como la especialización (economías de localización). Del lado de la NGE los trabajos 
empíricos han utilizado diversas medidas para captar la accesibilidad a los mercados, como el potencial de mercado propuesto por Redding y Venables (2004), el potencial real de mercado de Head y Mayer (2006) o el potencial externo de mercado que aparece en Mion (2004) y Hanson (2005). En virtud de que la información es necesaria para construir estas métricas no se encuentra disponible, ${ }^{10}$ usamos como medida de accesibilidad la distancia de cada área a la frontera más cercana con Estados Unidos. Consideramos que esta variable es representativa de la accesibilidad que tienen los productos manufactureros por dos razones. La primera es que de acuerdo a la información de la Administración General de Aduanas de México, más de 80\% de los productos manufacturados que se venden al exterior tienen como destino a los estadounidenses y la segunda es que gran parte de ese comercio se realiza utilizando como medio de transportación el transporte carretero. Por lo anterior, la segunda ecuación a estimar es la siguiente:

$$
f_{i j}=\varphi_{0}+\varphi_{1} \operatorname{lnden}_{j}+\varphi_{2} \operatorname{lndiv}_{j}+\varphi_{3} \operatorname{lnespc}_{i j}+\varphi_{4} \operatorname{lndistf}_{i j}+\varphi_{5} \varphi_{j}+\varphi_{i j}[2]
$$

En cual expresamos las covariables en logaritmos para interpretar los parámetros en términos de elasticidades y donde es la densidad en el área j, es la diversidad en el área j, capta la especialización en la industria i del área $\mathrm{j}$, es la distancia física de la industria i del área $\mathrm{j}$ hacia la frontera norte con Estados Unidos y es un vector de variables dummies de industria.

La medida densidad que usamos es parecida a la utilizada por Combes (2000).

$$
\text { den }_{j}=\frac{\text { empleo }_{j}}{\text { superficie }_{j}}
$$

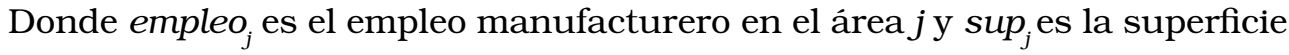
en kilómetros cuadrados del área $j$. El cómputo de esta medida, sin embargo tiene inconvenientes asociados con la geografía del territorio. En México los municipios exhiben una alta heterogeneidad en su extensión terrestre; en el sur del país existen muchos municipios con una superficie pequeña, en cambio en el norte se registran pocos municipios con una amplia extensión territorial. Para tener una medición comparable entre las diversas ubicaciones y que no se vea afectada por la heterogeneidad del territorio, utilizamos como denominador a la superficie neta definida como la extensión territorial

${ }^{10}$ Para el cómputo de estas medidas son necesarios distintos flujos de comercio para cada una de las áreas, como los flujos de comercio internacionales, los flujos de comercio entre áreas y los flujos comerciales al interior de las unidades territoriales. 
que no considera el espacio correspondiente a agricultura, pastizales, bosque, selva y matorrales. Para la especialización tomamos la medida mencionada en Combes (2000).

$$
e s p_{i j}=\left[\frac{E_{i j} / E_{j}}{E_{i} / E}\right]
$$

Donde es el empleo en la industria manufacturera i en el área j, es el empleo manufacturero total en el área j, es el empleo en la industria i y es el empleo manufacturero total en México. Para la medida de diversidad usamos la inversa del índice de Hirschman-Herfindahl como en Duranton y Puga (2000).

$$
d i v_{j}=\frac{1}{\sum_{i}^{n}\left(\frac{E_{i j}}{E_{j}}\right)^{2}}
$$

Para mediar accesibilidad a Estados Unidos utilizamos un indicador parecido al propuesto por Hanson (1998):

$$
\operatorname{distf}_{i j}=\frac{d_{j}}{\sum_{i}^{n}\left(\frac{E_{i j}}{E_{j}}\right)^{2} * d j}
$$

Donde $d_{j}$ es la distancia del área j al punto más cercano a la frontera con Estados Unidos.

La unidad espacial de análisis son las áreas que comprenden los mercados laborales locales (MLLs) de México. Esta clasificación proviene de Casado (2007) y fue elaborada aplicando una adaptación del algoritmo propuesto por Coombes et al. (1986) a los datos del Censo de Población y Vivienda (CpyV) del año 2000. Esta clasificación agrupa a los 2443 municipios vigentes en ese año en 415 MLLs de acuerdo a criterios de auto-contención o autonomía (desplazamientos residencia-trabajo) y de tamaño (número de trabajadores) entre municipios. La elección de esta unidad observación es relevante, ya que el uso de una unidad territorial construida con base en desplazamientos de los individuos por motivos de trabajo puede captar de mejor manera los efectos de la aglomeración sobre los salarios. 
Economía Informa núm. 381 julio - agosto • 2013 ㅂ ㅁ |

\section{III.2 Datos}

Los datos que usamos en este documento son los microdatos provenientes de la muestra aleatoria de 1\% del CPyV del año 2000, levantado por el Instituto Nacional de Estadística y Geografía (INEGI). Esta fuente contiene información detallada sobre ingresos, nivel educativo, edad, género, ocupación, municipio de residencia y de trabajo de la población en México. De esta información consideramos únicamente a los individuos varones asalariados cuya edad estuviera comprendida entre 15 a 65 años y que laboraran en alguna de las industrias comprendidas dentro del sector manufacturero conforme al Sistema de Clasificación Industrial de América del Norte México (SCIAN). ${ }^{11}$

La medida de salarios que usamos corresponde al ingreso por trabajo, la cual se compone de los ingresos por concepto de ganancia, comisión, sueldo, salario, jornal, propina o cualquier otro devengado de su participación en alguna actividad económica y que el INEGI expresa de manera mensual.

Para la estimación de la primera etapa en la base censal se identificaron los MLLs donde laboran los trabajadores manufactureros con base a la clasificación de Casado (2007). Esta base se restringió eliminado aquellos MLLs donde el número de trabajadores fuera menor a 50 observaciones, por lo cual la base en esta fase abarcó una muestra de más de 250 mil trabajadores.

Las variables de accesibilidad, economías de localización y de urbanización que usamos en este documento fueron construidas al nivel de MLLs. En su elaboración se utilizaron los datos del personal ocupado provenientes de los censos económicos de 1999 levantado por el INEGI. Asimismo, las distancias entre cada área y la frontera más cercana a Estados Unidios se calcularon mediante el uso de los mapas digitales del marco geoestadístico municipal del año de 2000 elaborado por el INEGI y la aplicación de diversos procedimientos en el software de información geográfica ArcGis. Para el cómputo de la superficie neta se utilizaron cifras de uso de suelo a nivel municipal correspondientes al año 2000, las cuales provienen del INEGI.

Para la base utilizada en la segunda fase se realizo un match entre los efectos fijos MLLs/industria obtenidos en la primera y las medidas de variables de accesibilidad, economías de localización y de urbanización computadas al nivel de MLLs, los observaciones que hicieron un match imperfecto no fueron consideradas. En el cuadro 1 se describe la estadística descriptiva de las covariables mencionadas.

\footnotetext{
${ }^{11}$ El scian también es comparable con la Clasificación Industrial Internacional Uniforme de todas las actividades económicas, Revisión 3 (CIIU-3), de la Organización de las Naciones Unidas, que es el clasificador de referencia para los clasificadores de actividades de la mayoría de los países.
} 


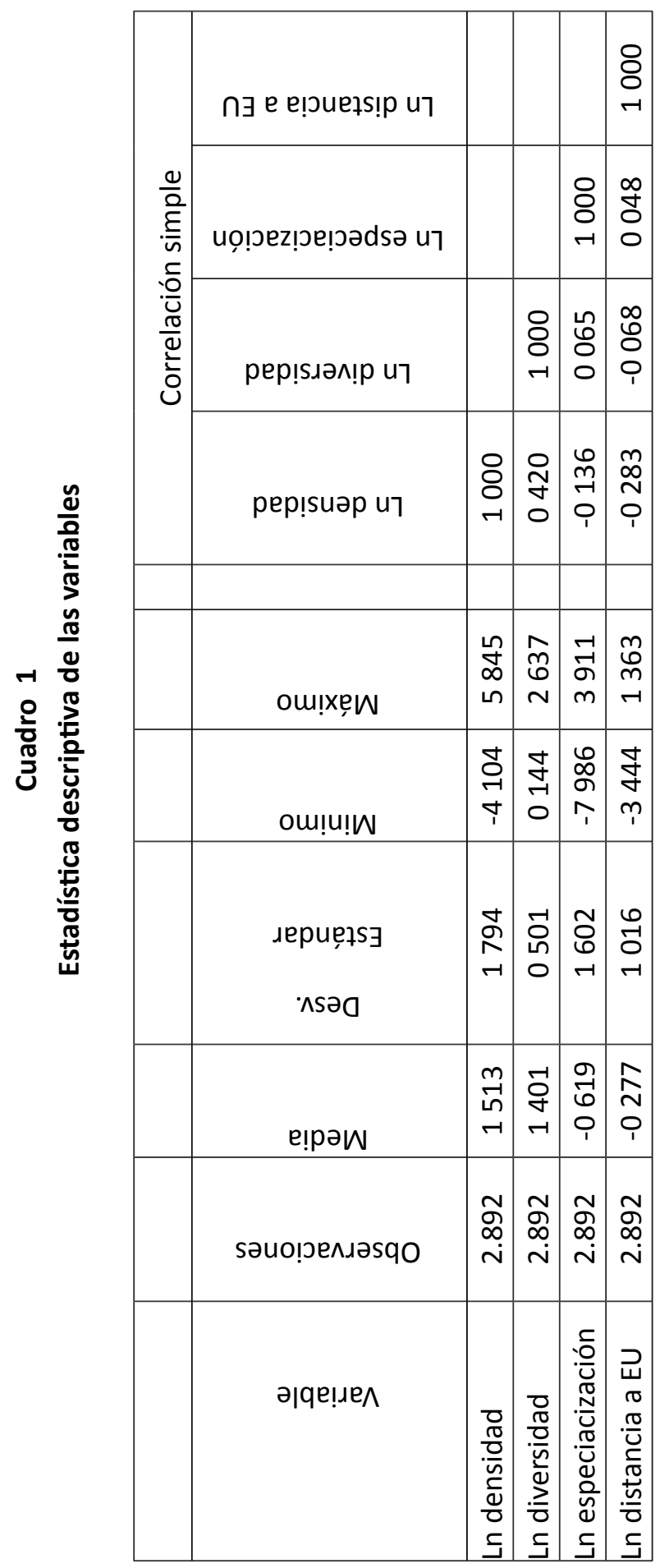




\section{Resultados}

\section{IV.1 Estimación en dos etapas}

De acuerdo a la estrategia empírica estimamos la ecuación [1] la cual relaciona el logaritmo del ingreso en función de la experiencia (edad y edad al cuadrado), seis niveles de educación (donde la categoría de referencia es la nula instrucción educativa) y efectos fijos MLLs/industria. Los resultados obtenidos se reportan en la columna uno del cuadro 2. Los parámetros obtenidos para todas las características individuales son altamente significativos a un nivel de $1 \%$. La relación entre edad y edad al cuadrado (proxy de experiencia) con respecto a los salarios describen un patrón en forma de $\mathrm{U}$ invertida, el cual refleja que a mayor experiencia de los individuos mayor será su salario, sin embargo, llega un momento en que la acumulación de mayor experiencia no se reflejará en un aumento de salario. Asimismo, los hallazgos muestran que los salarios son mayores conforme se aumentan los niveles de instrucción educativa. Por ejemplo, un trabajador con una instrucción de primaria tiene un salario 13\% mayor que un trabajador sin instrucción con el mismo nivel de experiencia. Por su parte un empleado manufacturero con un nivel de estudios de Licenciatura obtiene un salario $48 \%$ superior que un empleado sin instrucción con experiencia similar.

En la columna dos del cuadro 2 controlamos en la estimación por efectos fijos de ocupación, la cual es otra característica observable que tenemos de los trabajadores. La inclusión de estos controles disminuye la potencia de los parámetros estimados, sin dejar de perder su alta significatividad. Asimismo, esta estimación refleja un incremento del estadístico R2 al explicar $36 \%$ de la variación de los salarios contra $27 \%$ de la regresión previa.

Para la estimación de la ecuación [2] usamos los efectos fijos calculados en la estimación reportada en la columna dos del cuadro 2, así como las medidas computadas de acuerdo a lo descrito en la sección III. La tabla 3 contiene los resultados de la estimación de la segunda etapa. Por motivos de comparación incluimos paulatinamente cada una de las covariables consideradas. La columna uno presenta el coeficiente obtenido al considerar como variable independiente al logaritmo de la densidad, los resultados muestran una elasticidad de 0.0501 , este resultado se encuentra en el rango del obtenido por otros trabajos empíricos (Rosenthal y Strange, 2004), asimismo, este hallazgo indica que los MLLs con una mayor densidad de trabajadores manufactureros se encuentran asociados con niveles salaria- 


\section{Cuadro 2}

Estimación primera etapa

\begin{tabular}{|c|c|c|}
\hline & $(1)$ & $(2)$ \\
\hline \multicolumn{3}{|c|}{ Variable dependiente: Logaritmo de los salarios } \\
\hline \multirow[t]{2}{*}{ edad } & $0.0600 * *$ & $0.0539 * *$ \\
\hline & $(0.0010)$ & $(0.0010)$ \\
\hline \multirow[t]{2}{*}{ edad2 } & $-0.000670 * *$ & $-0.000621 * *$ \\
\hline & $(0.00001)$ & $(0.00001)$ \\
\hline \multicolumn{3}{|c|}{ Nivel de referencia ( $\sin$ instrucción = 0) } \\
\hline \multirow[t]{2}{*}{ Primaria } & $0.137^{* *}$ & $0.109 * *$ \\
\hline & $(0.0206)$ & $(0.0198)$ \\
\hline \multirow[t]{2}{*}{ Secundaria } & $0.196 * *$ & $0.162 * *$ \\
\hline & $(0.0082)$ & $(0.0077)$ \\
\hline \multirow[t]{2}{*}{ Bachillerato } & $0.320 * *$ & $0.257^{* *}$ \\
\hline & $(0.0106)$ & $(0.0096)$ \\
\hline \multirow[t]{2}{*}{ Licenciatura } & $0.483^{* *}$ & $0.368 * *$ \\
\hline & $(0.0134)$ & $(0.0114)$ \\
\hline \multirow[t]{2}{*}{ Posgrado } & $0.984 * *$ & $0.631 * *$ \\
\hline & $(0.0213)$ & $(0.0151)$ \\
\hline \multicolumn{3}{|l|}{ Efectos fijos } \\
\hline Mlls/industria & $\mathrm{Si}$ & $\mathrm{Si}$ \\
\hline Ocupación & No & $\mathrm{Si}$ \\
\hline $\mathrm{R} 2$ & 0.275 & 0.364 \\
\hline F-test & 1043.8 & 858.9 \\
\hline Observaciones & 256,114 & 256,114 \\
\hline
\end{tabular}

Nota: **, *, +, indican significancia a 1, 5 y 10\%, respectivamente. Entre paréntesis se reportan los errores estándar robustos.

les mayores que áreas con una menor densidad. En la columna dos observamos que el parámetro del logaritmo de la diversidad es positivo y altamente significativo, con lo cual soporta el supuesto de que las empresas se benefician de las externalidades que surgen de localizarse en mercados laborales con una mayor diversidad, permitiéndoles ofrecer salarios más altos.

Respecto a la variable de especialización encontramos un coeficiente negativo y estadísticamente no significativo (columna 3), con lo cual no encontramos evidencia de la existencia de economías de localización entre los 
MLLs. En contraparte la elasticidad de la distancia a Estados Uniidos registra un parámetro de -0.106 (columna 4), el cual es significativo a 1\%. De manera que si la distancia entre un mercado laboral y la frontera norte se incrementa en $10 \%$ los salarios en esa ubicación reflejara una disminución de $1.06 \%$. Este resultado se encuentra en consonancia a lo reportado por Hanson (1997), a pesar que el tamaño del coeficiente es ligeramente menor.

La columna 5 del cuadro 3 muestra los coeficientes obtenidos de la regresión que incorpora las covariables que fueron significativas en las cuatro estimaciones previas. Los resultados permiten caracterizar a los mercados laborales donde se retribuye de mejor manera a los trabajadores manufactureros, los cuales son los que registran una mayor densidad y diversidad económica, así como una menor distancia con respecto a Estados Unidos. Asimismo, esta caracterización nos permite explicar un 24\% de la desigualdad salarial de los trabajadores manufactureros atribuibles a las medidas de economías de aglomeración y de accesibilidad utilizada. Esta última regresión representa nuestra estimación base que usaremos como comparación en las siguientes secciones.

Finalmente, como prueba de robustez de la regresión base estimamos la ecuación [1] y [2] en una sola etapa, es decir, en la especificación de la primera ecuación incorporamos las medidas de economía urbana y de NGE, controlando por efectos fijos de industria, mercados laborales y de ocupación. Asimismo, corregimos los errores estándar clusterizando al nivel de mercado laboral siguiendo a Moulton (1986 y 1990). ${ }^{12}$ En la columna 6 de la tabla 3 se muestra que los resultados obtenidos de la estimación en una sola etapa son muy parecidos a los alcanzados en la estimación base.

\section{IV.2 Endogeneidad y dependencia espacial}

Un aspecto relevante con respecto a la estimación de la ecuación [2] tiene que ver con el problema de endogeneidad que surge entre la simultaneidad entre el salario y la densidad, territorios donde se paga a los trabajadores manufactureros con altos ingresos tiende a atraer a más obreros haciendo que estas áreas más aglomeradas. La presencia de esta endogeneidad ocasiona que nuestras estimaciones por MCO lleguen a ser sesgadas e inconsistentes.

${ }^{12}$ Este autor muestra que cuando micro datos son regresionados con respecto a variables agregadas, los errores estándar obtenidos por MCO son subestimados debido a que no se toma en cuenta la correlación que existe entre individuos dentro de la agrupación (cluster) a la cual hace referencia la variable agregada. 


\section{Cuadro 3}

\section{Estimación segunda etapa}

$\begin{array}{llllll}(1) & (2) & (3) & (4) & \text { (5) } & \text { (6) a/ }\end{array}$

Variable dependiente: Efectos fijos de mercado laboral/industria

\begin{tabular}{|c|c|c|c|c|c|c|}
\hline $\begin{array}{l}\text { ln densi- } \\
\text { dad }\end{array}$ & $\begin{array}{r}0.0501^{* *} \\
(0.0034) \\
\end{array}$ & & & & $\begin{array}{c}0.0233^{* *} \\
(0.0038) \\
\end{array}$ & $\begin{array}{r}0.0223^{* *} \\
(0.0008) \\
\end{array}$ \\
\hline $\begin{array}{l}\text { ln diver- } \\
\text { sidad }\end{array}$ & & $\begin{array}{c}0.148^{* *} \\
(0.0113) \\
\end{array}$ & & & $\begin{array}{c}0.104^{* *} \\
(0.0120) \\
\end{array}$ & $\begin{array}{r}0.117^{* *} \\
(0.0034) \\
\end{array}$ \\
\hline $\begin{array}{l}\text { ln espe- } \\
\text { cializa- } \\
\text { ción }\end{array}$ & & & $\begin{array}{r}-0.00082 \\
(0.0041)\end{array}$ & & & \\
\hline $\begin{array}{l}\text { ln dis- } \\
\text { tancia a } \\
\text { EU }\end{array}$ & & & & $\begin{array}{c}-0.106^{* *} \\
(0.0051)\end{array}$ & $\begin{array}{r}-0.0910^{* *} \\
(0.0051) \\
\end{array}$ & $\begin{array}{c}-0.107^{* *} \\
(0.0021) \\
\end{array}$ \\
\hline $\begin{array}{l}\text { Cons- } \\
\text { tante }\end{array}$ & $\begin{array}{r}6.934^{* *} \\
(0.0151) \\
\end{array}$ & $\begin{array}{r}6.795^{* *} \\
(0.0215) \\
\end{array}$ & $\begin{array}{r}6.982^{* *} \\
(0.0164) \\
\end{array}$ & $\begin{array}{r}6.951^{* *} \\
(0.0146) \\
\end{array}$ & $\begin{array}{r}6.801^{* *} \\
(0.0199) \\
\end{array}$ & $\begin{array}{r}6.606^{* *} \\
(0.0449) \\
\end{array}$ \\
\hline \multicolumn{7}{|l|}{$\begin{array}{l}\text { Efectos } \\
\text { fijos }\end{array}$} \\
\hline $\begin{array}{l}\text { Indus- } \\
\text { tria }\end{array}$ & $\mathrm{Si}$ & $\mathrm{Si}$ & $\mathrm{Si}$ & $\mathrm{Si}$ & $\mathrm{Si}$ & $\mathrm{Si}$ \\
\hline R2 & 0.164 & 0.145 & 0.102 & 0.198 & 0.244 & 0.476 \\
\hline F-test & 34.70 & 30.95 & 22.29 & 45.56 & 54.12 & 50.20 \\
\hline $\begin{array}{l}\text { Obser- } \\
\text { vaciones }\end{array}$ & 2892 & 2892 & 2892 & 2892 & 2892 & 255373 \\
\hline
\end{tabular}

Nota: **, *, +, indican significancia a 1,5 y $10 \%$, respectivamente. Entre paréntesis se reportan los errores estándar robustos.

a/ Estimación en una sola etapa de las ecuaciones [1] y [2]. Incorpora además efectos fijos de mercados laborales y de ocupación.

Los errores estándar se corrigen clusterizando al nivel de mercado laboral. 
Para tratar este asunto incorporamos en la estimación base la aproximación por variables instrumentales (VI). El método de variables instrumentales permite una estimación consistente cuando las covariables sufren del problema de endogeneidad y se basa en la elección de una variable (instrumento) que no pertenece al modelo inicialmente estimado, la cual debe estar correlacionada con la variable explicativa (endógena) y no estar correlacionada con el término de error de la ecuación estimada. Además, para que la elección de la VI sea un instrumento válido para tratar el problema de endogeneidad, la VI debe ser creíble desde el punto de vista económico y la ecuación a estimar debe estar correctamente identificada (Murray, 2006).

Como VI de la covariable de densidad ${ }^{13}$ se seleccionó a la red ferroviaria existente en México hasta el año de $1890 .{ }^{14} \mathrm{El}$ uso de esta variable se fundamenta en virtud de que la construcción de esta red ferroviaria permitió el traslado de personas y mercancías del centro y sur del país con la frontera de Estados Unidos, lo cual propició el nacimiento de nuevas urbes, así como un incremento en el número de habitantes de ciudades que a la fecha se mantienen (Kuntz y Riguzzi, 1996).

La VI se definió como una variable dicotómica, la cual toma el valor de 1 cuando MLL es un punto de inicio o fin de recorrido de la red, ${ }^{15} \mathrm{o}$ si es un nodo que enlazaba las diferentes redes. En la columna 1 del cuadro 4 replicamos los resultados de la estimación base y en la siguiente columna se reportan los resultados utilizando VI. El signo de los coeficientes apunta en la misma dirección de los resultados previos, a saber, los salarios son más altos en aquellos mercados laborales con gran diversidad económica, con

\footnotetext{
${ }^{13}$ De igual forma, este problema de endogeneidad lo encontramos en la relación entre salarios y diversidad económica, donde áreas con una alta diversidad que paga mayores salarios tiende a atraer más trabajadores, lo cual incrementa a su vez la diversidad del área. Lo ideal es instrumentar tanto la variable de densidad como la variable de diversidad, sin embargo, la elección de dos VI que sean instrumentos validos no es trivial. Debido a que ambas variables son más relevantes en ambientes aglomerados, consideramos que al instrumentar a la densidad también aligera el problema de endogeneidad en la variable de diversidad.

${ }^{14}$ Existen otras variables que en la literatura han sido utilizadas para solventar el problema de endogeneidad. Ciccone y Hall (1996) y Ciccone (2002) usan como instrumentos a variables históricas de densidad de población. Por su parte, Combes et al. (2009) consideran variables construidas con base a la estructura geológica de las unidades territoriales. No obstante, dada la no disponibilidad de esta información a nivel municipal imposibilitó su construcción.

${ }^{15}$ Las rutas que consideramos constituyen la columna vertebral del sistema ferroviario actual de México. La primera abarca el recorrido del Veracruz hasta la Ciudad de México con ramales en Puebla y Xalapa; la segunda es la que iba de la capital del país al Paso del Norte (hoy Ciudad Juárez) pasando por las ciudades de Irapuato, Silao, León, Aguascalientes, Zacatecas, Torreón y Chihuahua, con dos ramales, el de Silao a Guanajuato y el otro que llegaría a GuadalajaraManzanillo; y la última es la que unía a la Ciudad de México con Nuevo Laredo.
} 
una mayor densidad de trabajadores manufactureros y cercanos a la frontera con Estados Unidos. No obstante, el estimador de la densidad encuentra un aumento en la elasticidad de 0.0233 a 0.0419 y una disminución en la significancia de 1 a $5 \%$. Por su parte, los coeficientes de la diversidad y de la distancia mostraron un menor tamaño sin registrar ningún cambio en su significatividad. Angrist y Krueger (2000) mencionan que la estimación por VI no sólo corrige el problema de endogeneidad, sino también por un posible problema de error de medición en la variable endógena, lo que llevaría a un sesgo de atenuación en las estimaciones de MCO.

En el tratamiento del problema de la endogeneidad existe aspecto importante, el cual consiste en asegurar que la variable elegida para su corrección no sea un instrumento débil. ${ }^{16}$ Para abonar sobre este punto examinamos el estadístico F de Cragg-Donald Wald. En caso de que la prueba F sea mayor a 10 el instrumento no es considerado débil (Stock y Yogo, 2005). Al final de la columna dos del cuadro 4 notamos que el valor del estadístico $\mathrm{F}$ es de 102.8 lo cual muestra que la variable considerada como instrumento predice correctamente a la variable endógena.

En años recientes se ha puesto de manifiesto la importancia de tomar en cuenta la dependencia o autocorrelación espacial que puede surgir debido a que el valor de una variable en un lugar en el espacio está relacionado con el valor de otro u otros lugares en el espacio. El estimar por MCO ignorando este hecho ocasiona que los estimadores obtenidos sean ineficientes, la varianza residual será sesgada y las predicciones MCO sean ineficientes, generando un sesgo en la inferencia de los test de significación (Anselin, 1988).

Para solucionar este inconveniente Anselin (1988), propone incorporar los efectos espaciales dentro de la estimación, mediante el uso de matriz de pesos que refleja la interdependencia entre las unidades espaciales. Tradicionalmente, se hace uso de una matriz que contiene en sus elementos la inversa de las distancias entre las unidades espaciales elevadas al cuadrado y que es estandarizada dividiendo cada elemento de por la suma total de la fila a la que pertenece, de tal forma que la suma de cada fila de la matriz es igual a la unidad. A partir de esta matriz de pesos la presencia de la dependencia espacial (en el término de error o en la variable dependiente)

\footnotetext{
${ }^{16}$ En el caso de que el instrumento sea débil, la ligera presencia de correlación entre el instrumento y el término de error en la ecuación original puede ocasionar grandes inconsistencias en los coeficientes computados. Este problema surge en el momento cuando el instrumento esta correlacionado muy débilmente con el regresor (endógeno) o su tamaño es muy grande (Angrist y Krueger, 2001).
} 
puede ser controlada dentro de una estimación por Máximo Verosimilitud. Un inconveniente en el uso de la matriz de pesos es que no existe un conceso sobre su construcción. Además, en caso que optáramos por el uso de un forma de matriz en específico los requerimientos computacionales para estimar un modelo que tome en cuenta la dependencia espacial de nuestras observaciones es muy alto, en virtud de que implicaría el uso de una matriz con una dimensión de aproximadamente de 200 mil por 200 mil para la estimación de la primera etapa.

Para atender el asunto de la dependencia espacial y superar las limitaciones informáticas seguimos un procedimiento parecido al descrito en Rodríguez-Posé (1999), que utiliza variables ponderadas por la media nacional para aliviar el problema de autocorrelación espacial. ${ }^{17}$ En la columna 3 del cuadro 4 se reportan los coeficientes estimados siguiendo el procedimiento mencionado. Los resultados son muy similares a los obtenidos cuando utilizamos VI, un aumento en el tamaño de la elasticidad de la densidad y una disminución en la magnitud de las elasticidades de la diversidad y la distancia a Estados Unidos. Estos hallazgos muestran que una vez descontados los efectos de dependencia espacial en nuestras unidades de observación se mantienen los resultados de nuestra estimación base. Finalmente, en la columna cuatro del cuadro 4 se reportan los parámetros obtenidos de estimar por VI y ocupar el procedimiento de Rodríguez-Posé (1999) para atender el problema de autocorrelación espacial. Las elasticidades estimadas son bastante cercanas a las reportadas en la estimación base (ver cuadro 4).

\section{IV.3 Ámbito de influencia de las economías de aglomeración}

En esta parte abordamos la cuestión sobre el ámbito de influencia de nuestras medidas de economías de aglomeración sobre las desiguladades salariales en México, es decir, analizamos a que nivel geográfico los efectos (en magnitud y significancia) de la densidad, diversidad y la distancia a Estados Unidos, explican las diferencias de salarios en México después de controlar la heterogeneidad de las características individuales de los trabajadores.

Para abordar este asunto, aplicamos nuevamente el procedimiento en dos etapas para estimar las ecuaciones [1] y [2]. Primero, se realiza la regresión de la ecuación [1] tanto al nivel de entidad federativa como de regio-

${ }^{17}$ Nuestras variables expresadas en logaritmos primero son ponderadas por la media nacional y después las expresamos en logaritmos. 


\section{Cuadro 4}

Estimaciones por VI y para atender dependencia espacial

\begin{tabular}{|c|c|c|c|c|}
\hline & (1) & $(2)$ & (3) & (4) \\
\hline \multicolumn{5}{|c|}{ Variable dependiente: Efectos fijos de mercado laboral/industria } \\
\hline \multirow[t]{2}{*}{ In densidad } & $0.0233 * *$ & $0.0419 *$ & $0.0442 * *$ & $0.0305^{*}$ \\
\hline & $(0.0038)$ & $(0.0190)$ & $(0.0044)$ & $(0.0136)$ \\
\hline \multirow[t]{2}{*}{ In diversidad } & $0.104 * *$ & $0.0807^{* *}$ & $0.0769 * *$ & $0.0946 * *$ \\
\hline & $(0.0120)$ & $(0.0277)$ & $(0.0119)$ & $(0.0215)$ \\
\hline \multirow[t]{2}{*}{ In distancia a EU } & $-0.0910 * *$ & $-0.0824 * *$ & $-0.0792 * *$ & $-0.0861^{* *}$ \\
\hline & $(0.0051)$ & (0.0098) & $(0.0055)$ & $(0.0083)$ \\
\hline \multirow[t]{2}{*}{ Constante } & $6.801 * *$ & $6.815^{* *}$ & $-0.0874 * *$ & $-0.104 * *$ \\
\hline & (0.0199) & $(0.0253)$ & $(0.0147)$ & $(0.0211)$ \\
\hline \multicolumn{5}{|l|}{ Efectos fijos } \\
\hline Industria & Si & Si & Si & Si \\
\hline $\mathrm{R} 2$ & 0.244 & 0.238 & 0.258 & 0.256 \\
\hline F-test & 54.12 & 51.74 & 59.89 & 53.45 \\
\hline \multicolumn{5}{|c|}{ Test de sobre identificación } \\
\hline [P-Value] & & 0000 & & 0000 \\
\hline \multicolumn{5}{|c|}{ Test de debilidad de instrumentos } \\
\hline \multicolumn{5}{|l|}{ Cragg-Donald } \\
\hline Wald F statistic & & 102865 & & 324016 \\
\hline Observaciones & 2892 & 2892 & 2892 & 2892 \\
\hline
\end{tabular}


nes ${ }^{18}$ y posteriormente para la ecuación [2] los efectos fijos computados se regresionan con respecto a las medidas de economías de aglomeración que utilizamos y que son construidas a esas escalas.

En la columna 1 del cuadro 5, se muestran los parámetros obtenidos en nuestra estimación base. En las columnas 2 y 3 se presentan los resultados de las regresiones utilizando a los estados y las regiones como unidad de análisis.

Los parámetros de la elasticidad de la densidad y de la diversidad económica no resultaron ser significativos para explicar a una escala estatal ni regional las diferencias salariales a esos niveles. Estos resultados sugieren que los beneficios derivados por la concentración de la actividad económica se encuentran ubicados en territorios de menor escala territorial.

Por su parte, el coeficiente del costo de transporte a la frontera norte, resulta ser significativo y creciente conforme nos movemos, a una escala espacial mayor. Al pasar de -0.091 al nivel de mercados laborales a -0.209 en regiones. Asimismo, la comparación de los coeficientes de la distancia a diferentes nos permite dilucidar una clara diferencia salarial entre el norte y el sur del país. El resultado en la estimación base apuntaba a que entre más grande fuera la distancia de los MLLs con respecto a los estadounidenses o menor acceso tuviera a dicho mercado, los salarios serían menores. Cuando se considera los hallazgos a nivel de entidades federativas el efecto de la elasticidad es mayor exhibiendo diferencias entre los salarios promedio que se pagan en los estados, la estimación a esta escala explica $42 \%$ de la variación salarial. Al considerar la dimensión regional encontramos que las regiones que hacen frontera con Estados Unidos o con mayor acceso a ese mercado son que las reportarían mejores salarios, en contra parte, las ubicadas al sur del país serían las que menor paga registrarian, estas diferencias son explicadas por nuestra estimación en 66 por ciento.

Estos hallazgos se encuentra en la misma dirección de los descritos por Combes et al. (2008) y Fingleton (2006), en el sentido de que la aproximación de la NGE parece describir mejor el comportamiento de los salarios en mayores unidades geográficas, como lo son estados y regiones donde la distancia a los mercados (accesibilidad) parece tener un rol más importante.

${ }^{18}$ La región Centro-Este comprende a las entidades federativas del Distrito Federal, Estado de México, Hidalgo, Morelos, Puebla, Querétaro y Tlaxcala. La región Centro-Occidente comprende a los estados de Colima, Guanajuato, Jalisco, Michoacán, Zacatecas, Aguascalientes, Nayarit y San Luis Potosí. La región este abarca a los estados de Veracruz y Tabasco. La Noreste incluye a los estados de Nuevo León y Tamaulipas. La región Noroeste comprende a Baja California, Sonora, Baja California Sur y Sinaloa. La norte considera a Coahuila, Chihuahua y Durango. La región península considera a Campeche, Quintana Roo y Yucatán. Finalmente, en la región Sur se encuentran los estados de Chiapas, Guerrero y Oaxaca. 
Por otra parte, los efectos de las economías de aglomeración parece ser más relevante a escalas espaciales menores donde se espera que las externalidades jueguen un rol más preponderante.

\section{Conclusiones}

Las limitaciones de este trabajo son varias. Por un lado, las estimaciones no controlan las características no observables de los individuos, la cuales de acuerdo con los hallazgos de Combes, et al. (2008) explican una amplia parte de las desigualdades salariales y pueden reducir la magnitud de los efectos estimados de las variables de economía de aglomeración y de NEG utilizadas. Por otro lado, consideramos que el problema de endogenidad que se presenta en la variable de diversidad necesita ser más explorado considerando otras variables instrumentales. Estos inconvenientes podrían ser abordados me-

\section{Cuadro 5}

Ámbito de influencia de las economías de aglomeración

\begin{tabular}{lccr}
\hline & $(1)$ & $(2)$ & $(3)$ \\
\hline In densidad & \multicolumn{1}{c}{ MLL } & \multicolumn{1}{c}{ Estados } & \multicolumn{1}{c}{ Regiones } \\
& $0.0233^{* *}$ & 0.00212 & 0.00728 \\
& $(0.0038)$ & $(0.0059)$ & $(0.0120)$ \\
\hline In diversidad & $0.104^{* *}$ & -0.0147 & 0.0599 \\
& $(0.0120)$ & $(0.0263)$ & $(0.0508)$ \\
\hline In distancia a EU & $-0.0910^{* *}$ & $-0.141^{* *}$ & $-0.209^{* *}$ \\
& $(0.0051)$ & $(0.0061)$ & $(0.0195)$ \\
\hline Constante & $6.801^{* *}$ & $7.029^{* *}$ & $6.848^{* *}$ \\
& $(0.0199)$ & $(0.0502)$ & $(0.0968)$ \\
\hline
\end{tabular}

Efectos fijos

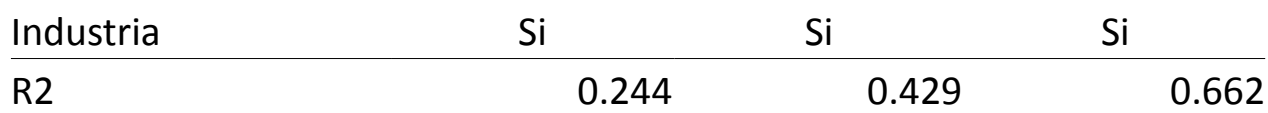

\begin{tabular}{lrrr} 
F-test & 54.12 & 36.37 & 11.19 \\
Observaciones & 2,892 & 665 & 147 \\
\hline
\end{tabular}

Nota: $* *, *,+$, indican significancia a 1,5 y $10 \%$, respectivamente. Entre paréntesis se reportan los errores estándar robustos. 
diante el uso de datos con una estructura de panel, lo cual se deja como un asunto de futura investigación. Asimismo, la estimación base se vería enriquecida al controlar otros aspectos que también se encuentran relacionados con la desigualdad salarial como la presencia de inversión extranjera en la firma (Feenstra y Hanson, 1997; Hanson y Harrison, 1999), las externalidades de capital humano y cercanía de fuentes naturales (Hanson, 2003), o el estatus de formalidad o informalidad del trabajador en el mercado laboral (Levy, 2008). Esto también se considera como parte de un trabajo futuro.

Este trabajo muestra que las medidas de densidad, diversidad y distancia a Estados Unidos (accesibilidad) resultaron significativas para explicar las diferencias salariales entre los mercados laborales de México. Los parámetros estimados son robustos a otro tipo de estimación, así como a problemas de endogeneidad y de dependencia espacial. Asimismo, encontramos que las economías de aglomeración tienen un impacto relevante para explicar las desigualdades salariales en unidades espaciales pequeñas, como el caso de los mercados laborales. Por el contrario, la medida de la NGE muestra describir de mejor manera las diferencias salariales en un ámbito territorial mayor, como en el caso de estado y regiones.

Asimismo, los resultados de la estimación base muestran que los mercados laborales que registran más altos salarios son aquellos con una alta densidad de trabajadores manufactureros, con mayor diversidad económica y que se encuentran a una distancia cercana de Estados Unidos. Para disminuir esta desigualdad utilizando las variables consideradas como instrumentos de política es posible sugerir que el fomento para la creación de clúster de empresas de distintos tipos en mercados labores con una baja actividad económica incrementaría la diversidad económica del área e incrementaría la densidad de trabajadores, con lo cual podrían generase externalidades derivadas de las economías de aglomeración.

Esta medida podría estar acompañada con mecanismos que coadyuvaran a mejorar la accesibilidad de los mercados laborales a los principales destino de consumo de los productos manufactureros. Una forma de incrementar la accesibilidad a Estados Unidos de los mercados laborales, es implementando planes de lógica que permitan optimizar las rutas con la infraestructura carretera disponible a fin de minimizar los costos de transporte de las mercancías enviadas a dicho país. Adicionalmente, las inversiones en infraestructura carretera deberían de estar enfocada a conectar a los mercados laborales más aislados con las principales rutas de acceso al mercado americano. Para los mercados laborales ubicados en el sur de país 
la accesibilidad podría además incrementarse mediante el uso del transporte marítimo.

\section{Bibliografía}

Angrist, Joshua y Alan, Krueger (2000), "Empirical Strategies in Labor Economics”, Handbook of Labor Economics 3A, Orley Ashenfelter y David Card, eds., pp. 1277-1282.

Angrist, Joshua y Alan, Krueger (2001), "Instrumental Variables and the Search for identification: From Supply and Demand to Natural Experiments", Journal of Economic Perspectives, vol. 15, núm. 4, pp. 69-85.

Anselin, Luc (1988), Spatial Econometrics, Kluwer, Dordrecht.

Ariola, Jim y Juhn, Chinhui (2005), "Wage Inequality in Post-Reform Mexico" IZA Discussion paper series, núm. 1525.

Brakman, Steven, Garretsen, Harry y Schramm, Marc (2004), "The Spatial Distribution of Wages and Employment: Estimating the Helpman-Hanson Model for Germany," Journal of Regional Science, Vol. 44(3), pp. 437-466.

Casado, José María (2007), Estructura regional de los mercados laborales locales en México. Tesis de Doctorado en Geografía, Facultad de Filosofía y Letras, UNAM, México.

Cañonero, Gustavo y Werner, Alejandro, (2002), "Salarios Relativos y Liberalización del Comercio en México”, El Trimestre Económico, vol. 69, núm. 273(1), pp. 123-142.

Chiquiar, Daniel (2008), "Globalization, regional wage differentials and the Stolper-Samuelson Theorem: Evidence from Mexico” Journal of International Economics, vol. 74(1), pp. 70-93.

Ciccone, Antonio (1997), “Technology Diffusion, Dynamic Externalities, and the Spatial Distribution of Wages in the US”, Mimeo, University of California, Berkeley.

Ciccone, Antonio (2002) “Agglomeration effects in Europe," European Economic Review, vol. 46, pp. 213-227.

Ciccone, Antonio y Hall, Robert (1996), "Productivity and the Density of Economic Activity". American Economic Review, vol. 86, pp. 54-70.

Combes, Pierre-Philippe (2000), "Economic structure and local growth: France, 1984-1993”, Journal of Urban Economics, vol. 47, pp. 329-355.

Combes, Pierre-Philippe, Duranton, Gilles y Gobillon, Laurent (2008), "Spatial wage disparities: Sorting matters!," Journal of Urban Economics, vol. 63(2), pp. 723-742. 
Combes, Pierre-Philippe, Duranton, Gilles, Gobillon, Laurent y Roux, Sébastien (2009), "Estimating agglomeration economies with history, geology, and worker effects," in Edward L. Glaeser (ed.), The Economics of Agglomeration.

Coombes, M.G., Green, A.E. y Openshaw, S. (1986), “An efficient algorithm to generate official statistical reporting areas: the case of the 1984 Travel-to-Work Areas”, Journal of the Operational Research Society, vol. 37, pp. 943-953.

Cragg, Michael Ian y Epelbaum, Mario (1996), "Why Has Wage Dispersion Grown in Mexico? Is it the Incidence of Reforms or the Growing Demand for Skills?", Journal of Development Economics, vol. 51, pp. 99-116.

Duranton, Gilles \& Puga, Diego (2000) "Diversity and specialisation in cities: Why, where and when does it matter?”, Urban Studies, vol. 37, núm. 3, pp. 533-555.

Duranton, Gilles y Puga, Diego (2004), "Micro-foundations of urban agglomeration economies," in Henderson, J.V. y Thisse, J.F. (Eds), Handbook of Regional and Urban Economics, vol. 4, pp. 2063-2117.

Esquivel, Gerardo y Rodríguez López, José A. (2003), “Technology, trade, and wage inequality in Mexico before and after NAFTA", Journal of Development Economics, vol. 72 (2), pp. 543-565.

Fally, Thibault, Paillacar, Rodrigo y Terra, Cristina (2010), "Economic geography and wages in Brazil: Evidence from micro-data", Journal of Development Economics, vol. 91(1), pp. 155-168.

Feenstra, Robert y Hanson, Gordon (1997) "Foreign Direct Investment and Relative Wages: Evidence from Mexico`s Maquiladoras.” Journal of International Economics Vol. 42, pp. 371-93.

Feliciano, Zadia (2001), "Workers and trade liberalization: the impact of trade reforms in Mexico on wages and employment", Industrial and Labor Relations Review, Vol 55, núm. 1, pp. 95-115.

Fingleton, Bernard (2006), "The new economic geography versus urban economics: an evaluation using local wage rates in Great Britain”, Oxford Economic Papers, vol. 58, pp. 501-530.

Hanson, Gordon (1996), "Localization Economies, Vertical Organization, and Trade”, The American Economic Review, Vol. 86, No. 5, pp. 1266-1278.

Hanson, Gordon (1997), "Increasing Returns, trade and the regional Structure of Wages”; The Economic Journal, vol. 107, núm. 440, pp. 113-133.

Hanson, Gordon (1998), "Regional Adjustment to Trade Liberalization”, $R e^{-}$ gional Science and Urban Economics, vol. 28, pp. 419-444. 
Hanson, Gordon (2003), "What Has Happened to Wages in Mexico since NAFTA?," NBER Working Papers 9563, National Bureau of Economic Research, Inc.

Hanson, Gordon y Harrison, Ann (1999), "Trade Liberalization and Wage Inequality in Mexico", Industrial and Labor Relations Review, vol. 52, núm. 2, pp. 271-288.

Hanson, Gordon (2005), "Market potential, increasing returns and geographic concentration”, Journal of International Economics, vol. 67, pp. 1-24.

Head, Keith y Mayer, Terry (2004), "The Empirics of agglomeration and trade", in Henderson, J.V. y Thisse, J.F. (Eds), Handbook of Regional and Urban Economics, vol. 4, Amsterdam, North-Holland.

Head, Keith y Mayer, Thierry (2006), "Regional Wage and Employment Responses to Market Potential in the EU," Regional Science and Urban Economics, vol. 36(5), pp. 573-594.

Henderson, J. Vernon (1986), "Efficiency of resource usage and city size," Journal of Urban Economics, Elsevier, vol. 19(1), pp. 47-70.

Hering, Laura y Poncet, Sandra (2010), "Market Access Impact on Individual Wage: Evidence from China", The Review of Economics and Statistics, vol. 92(1), pp. 145-159.

Hirschman, A. (1958), The Strategy of Economy Development (Yale University Press, New Haven, CT).

Krugman, Paul (1991a), "Increasing returns and Economic Geography", Journal of Political Economy, vol. 99, pp. 438-499.

Krugman, Paul (1991b), Geography and Trade, Cambridge, MA. MIT Press.

Kuntz, Sandra y Riguzzi, Paolo (1996), Ferrocarriles y vida económica en México, 1850-1950. Del surgimiento tardio al decaimiento precoz, El Colegio Mexiquense-UAM-Xochimilco-Ferrocarriles Nacionales de México, México.

Marshall, A. (1890), Principles of Economics: An Introductory, vol. 7th edition (Macmillan, London).

Meza, Liliana (1999), "Cambios en la estructura salarial de México en el periodo 1988-1993 y el aumento en el rendimiento de la educación superior", El Trimestre Económico, vol. LXVI (2), núm. 262, abril-junio, pp. 189-226.

Mion, Giordano (2004), "Spatial Externalities and Empirical Analysis: The Case of Italy”, Journal of Urban Economics, vol. 56(1), pp. 97-118.

Moulton, Brent R. (1986), "Random Group Effects and the Precision of Regression Estimates," Journal of Econometrics, vol. 32, núm.3, pp. 385-397.

Moulton, Brent R. (1990), “An Illustration of a Pitfall in Estimating the Effects of Aggregate Variables on Micro Unit," The Review of Economics and Statistics, vol. 72, núm. 2, pp. 334-38. 
Murray, Michael P. (2006), "The Bad, the Weak and the Ugly: Avoiding the Pitfalls of Instrumental Variables Estimation," Journal of Economic Perspectives, vol. 20, pp. 111-132.

Niebuhr, Annekatrin (2006), "Market access and regional disparities". The Annals of Regional Science, vol. 40, núm. 2, pp. 313-334.

Redding, Stephen y Venables, Anthony (2004), "Economic geography and international inequality”, Journal of International Economics, vol. 62, pp. 53-82.

Revenga, Ana, (1997), "Employment and Wage Effects of Trade Liberalization: The Case of Mexican Manufacturing”. Journal of Labor Economics, vol. 15, pp. 520- 543.

Robertson, Raymond (2004), "Relative prices and wage inequality: evidences from Mexico”, Journal of International Economic, vol. 64(2), pp. 387-409.

Rodríguez-Pose, Andrés (1999), Convergence or divergence? Types of Regional Responses to Socioeconomic Change”. Tijdschrift voor Economische en Sociale Geografie, vol. 90, pp. 363-378.

Rosenthal, Stuart S. y Strange, William C. (2004), "Evidence on the nature and sources of agglomeration economies" en Henderson, J.V. y Thisse, J.F. (Eds), Handbook of Regional and Urban Economics, vol. 4, Amsterdam, North-Holland, pp. 2119-2171.

Stock, J.H. y Yogo, M. (2005), "Testing for Weak Instruments in Linear IV Regression”. In D.W.K. Andrews and J.H. Stock, eds., Identification and Inference for Econometric Models: Essays in Honor of Thomas Rothenberg, Cambridge: Cambridge University Press, pp. 80-108.

Tan, Hong y Batra, Geeta (1995), "Technology and industry wage differentials: evidence from three developing countries”, PSD Occasional Paper, núm. 5, The World Bank.

Weber, A. (1909), Theory of the Location of Industries (University of Chicago Press, Chicago, IL). 